\title{
Conflictos semióticos sobre la correlación y regresión en los libros de texto de Bachillerato
}

\author{
María M. Gea Serrano, Universidad de Granada (España) \\ María del Mar López-Martín, Universidad de Granada (España) \\ Rafael Roa Guzmán, Universidad de Granada (España)
}

Recibido el 5 de mayo de 2015; aceptado el 2 de septiembre de 2015

\section{Conflictos semióticos sobre la correlación y regresión en los libros de texto de Bachillerato \\ Resumen}

El objetivo de este trabajo es caracterizar los conflictos semióticos que se presentan en el tema de correlación y regresión en los libros de texto españoles de Bachillerato. Para ello se realiza un análisis de contenido de los correspondientes capitulos en una muestra de 16 libros de texto de Bachillerato, ocho de la modalidad de Ciencia y Tecnología y ocho de la modalidad de Humanidades y Ciencias Sociales. Nos basamos en el Enfoque onto-semiótico del conocimiento y la instrucción matemática en didáctica de la matemática, en que se diferencia el significado institucional y personal de los objetos matemáticos y se define el conflicto semiótico como disparidad entre dichos significados. En nuestro análisis encontramos una amplia variedad de conflictos relacionados con el lenguaje utilizado, la definición de conceptos o presentación de propiedades y procedimientos, junto a algunos argumentos. Estos resultados advierten al profesor para evitar que dichos conflictos se manifiesten en el aprendizaje de los estudiantes. Finalmente, se proporcionan criterios para mejorar la presentación de la correlación y regresión en los textos de Bachillerato.

Palabras clave. Regresión y correlación; libros de texto; conflictos semióticos; Bachillerato.

\section{Conflitos semióticos sobre a correlação e regressão livros didáticos da Bacharelado .}

\section{Resumo}

O objetivo deste trabalho é caracterizar os conflitos semióticos que surgem sobre a questão da correlação e regressão no livros de texto Espanhol Bacharelado. Para fazer uma análise de conteúdo dos capítulos é realizada em uma amostra de 16 livros didáticos de Bacharelado, oito do modo de Ciência e Tecnologia e oito na forma de Ciências Humanas e Sociais. Contamos com a abordagem onto-semiótico do conhecimento matemático e instrução em educação matemática, que difere significado institucional e pessoal dos objetos matemáticos e conflitos semiótica é definida como a disparidade entre esses significados. Em nossa análise, encontramos uma grande variedade de conflitos relativos a linguagem utilizada, a definição de conceitos e apresentação de propriedades e métodos, juntamente com alguns argumentos. Estes resultados alertam o professor para evitar tais conflitos se manifestam na aprendizagem dos alunos. Finalmente, os critérios são fornecidos para melhorar a apresentação de correlação e de regressão textos Bacharelado .

Palavras chave. Regressão e correlação; livros didáticos; conflitos semióticos; Bacharelado.

Para citar: Gea, M. M., López-Martín, M. M., \& Roa, R. (2015). Conflictos semióticos sobre la correlación y regresión en los libros de texto de Bachillerato. Avances de Investigación en Educación Matemática, 8, 29 - 49.

(C) Sociedad Española de Investigación en Educación Matemática (SEIEM). www.seiem.es 
Semiotic conflicts related to correlation and regression in High School textbooks

\section{Abstract}

The aim of this work is to characterize the semiotic conflicts that arise on the topic of correlation and regression in the Spanish High School textbooks. A content analysis of the chapters dealing with this topic is performed on a sample of 16 high school textbooks, eight of the Science and Technology specialty and eight directed to Humanities and Social Sciences. We base on the onto-semiotic approach of mathematical knowledge and instruction in mathematics education that discriminates between institutional and personal meaning of mathematical objects; semiotic conflict in this framework is defined as a disparity between these two meanings. In our analysis we found a wide variety of conflicts concerning the language used, the definition of concepts and presentation of properties and methods, along with some arguments. These results alert the teacher to avoid that such conflicts are transmitted to the student learning. Finally, some criteria are provided to enhance the presentation of correlation and regression in the High School textbooks.

Key words. Regression and correlation; textbooks; semiotic conflicts; High School.

\section{Conflits sémiotiques sur la corrélation et la régression dans les manuels de Baccalauréat.}

\section{Résumé}

Le but de ce travail est de caractériser les conflits sémiotiques qui se presenten sur la question de la corrélation et de régression dans les manuels espagnols de Baccalauréat. Une analyse de contenu des chapitres est effectuée sur un échantillon de 16 manuels scolaires, huit de la spécialité de la science et de la technologie et huit de la spécialité de sciences humaines et sociales. Nous comptons sur l'approche onto-sémiotique des connaissances mathématiques et l'enseignement dans l'enseignement des mathématiques, il diffère institutionnel et personnel signifié d'objets mathématiques; le conflit sémiotique est défini comme la disparité entre ces significations. Dans notre analyse, nous trouvons une grande variété de conflicts concernant la langage utilisée, la définition des concepts et la présentation des propriétés et méthodes, avec quelques arguments. Ces résultats alertent l'enseignant à éviter que tels conflits se manifestent dans l'apprentissage des élèves. Enfin, quelques critères sont fournis pour améliorer la présentation de corrélation et de régression dans les textes de Baccalauréat.

Paroles clés. Régression et corrélation; manuels; conflits sémiotiques; Baccalauréat.

\section{Introducción}

La correlación y regresión son dos ideas fundamentales en estadística, puesto que constituyen la base de multitud de métodos estadísticos (Engel \& Sedlmeier, 2011). Estos conceptos extienden la idea de dependencia funcional, por lo que su inclusión en el primer curso de Bachillerato de las modalidades de Ciencias y Tecnología y Humanidades y Ciencias Sociales es claramente justificada (MEC, 2007). Igualmente se incluye en el nuevo currículo de Bachillerato en las especialidades de Ciencias y Humanidades y Ciencias Sociales (MECD, 2014).

La enseñanza de este tema no es simple, pues la investigación ha descrito sesgos de razonamiento y dificultades de comprensión; por ejemplo, no apreciar la correlación inversa, tener un sentido determinista o local de la correlación o identificar correlación con causalidad (Estepa, 2008; Estepa \& Batanero, 1995; Zieffler \& Garfield, 2009). Dichas dificultades y creencias, en algunos casos, resisten al cambio incluso después de la enseñanza (Batanero, Estepa \& Godino, 1997). También se han observado errores al interpretar los coeficientes de correlación y regresión (Sánchez Cobo, 1999; Sánchez Cobo, Estepa \& Batanero, 2000; Truran, 1995).

El objetivo de este trabajo es analizar los posibles conflictos semióticos que pueden ser inducidos por la presentación de la correlación y regresión en los libros de 
texto del Bachillerato español. Complementa otros previos en que se han analizado diferentes objetos matemáticos utilizados en la presentación del tema en los mismos textos utilizados en este trabajo, como por ejemplo, las variables que definen los problemas utilizados para contextualizar la enseñanza de la correlación y regresión y que se describirán más adelante, en el apartado sobre antecedentes.

\section{Marco teórico}

Nos basamos en el Enfoque Onto-Semiótico del conocimiento y la instrucción matemática (EOS) (Godino, 2002; 2012; Godino \& Batanero, 1994; 1999; Godino, Batanero \& Font, 2007), que asume que los objetos matemáticos surgen de las prácticas matemáticas (acciones u operaciones) como respuesta a situaciones problemáticas extra o intra matemáticas. En este marco teórico el término "objeto matemático" tiene un significado muy amplio y puede ser considerado desde el punto de vista institucional (por ejemplo, una institución de enseñanza) o personal (de cada persona). Se clasifica a los objetos matemáticos en situación-problema, lenguaje, conceptos, proposiciones, procedimientos, y argumentos.

El significado de un objeto, sería el conjunto de prácticas matemáticas asociadas al campo de problemas de donde surge dicho objeto (en Gea, Batanero, Cañadas \& Contreras, 2013, encontramos un análisis detallado del campo de problemas asociados a la correlación y regresión) y que puede variar en distintas instituciones y por tanto, tiene un carácter relativo. De ello se deduce la importancia de analizar la enseñanza y los recursos utilizados en ella, como los libros de texto, ya que la progresiva construcción del significado personal del estudiante depende directamente de los objetos que se describan y apliquen en su quehacer matemático, y el libro de texto apoya la actividad del alumno.

Dada la diversidad de objetos, así como su dimensión ostensiva y no ostensiva, los autores analizan los procesos de semiosis que se realizan en el desarrollo de la actividad matemática. El análisis ontológico-semiótico propuesto por el EOS ofrece un instrumento para reflejar las relaciones entre objetos matemáticos (función semiótica) y los procesos interpretativos en las prácticas matemáticas (Godino, 2002; Godino et al., 2007). Cuando entre dos objetos matemáticos se establece una dependencia en la que uno de ellos se pone en lugar del otro, o bien uno es usado por otro, uno de los objetos matemáticos adquiere el estatus de significante y el otro de significado (Godino et al., 2007).

El EOS postula que, la expresión y el contenido pueden ser cualquier tipo de objeto y la relación expresión-contenido no se limita a la acepción representación (Font, Godino \& D'Amore, 2007). Atendiendo a las relaciones de dependencia entre la expresión y el contenido se pueden distinguir tres tipos de funciones semióticas (D'Amore \& Godino, 2007; Godino, Font, Contreras \& Wilhelmi, 2006): 1) cuando un objeto se pone en lugar de otro para un cierto propósito hablamos de función semiótica representacional; 2) si un objeto utiliza a otro u otros como instrumento hablamos de función semiótica instrumental; 3) si dos o más objetos componen un sistema del cual emergen nuevos objetos hablamos de función semiótica estructural.

El alumno activa su propia trama o red/redes de funciones semióticas pudiendo reducir o aumentar la complejidad de dicha trama, y por otra parte, facilitar la realización, de un modo efectivo, de la tarea propuesta (Godino \& Batanero, 1994). 
Las prácticas que desarrolla el alumno serán consideradas correctas si se conforman a la institución. Surge así la noción de conflicto semiótico para tratar cualquier:

Disparidad o discordancia entre los significados atribuidos a una expresión por dos sujetos (personas o instituciones) en interacción comunicativa y pueden explicar las dificultades y limitaciones de los aprendizajes y las enseñanzas implementadas (Godino, 2002, p.250).

Estas interpretaciones o representaciones incorrectas desde el punto de vista institucional (conflictos semióticos), pueden llevar a producir errores durante la resolución de problemas, que no son debidos a falta de conocimiento, sino a no haber relacionado adecuadamente los dos términos de una función semiótica. Por tanto, los conflictos semióticos explicarían algunos errores de los estudiantes. Nuestro trabajo se orienta a identificar la presencia de posibles conflictos semióticos explícitos o implícitos en los libros de texto analizados. La importancia de su identificación es que dichos conflictos pudieran reproducirse posteriormente en los estudiantes que interpretan y aplican el libro de texto y causar errores en su aprendizaje. En lo que sigue se presentan los antecedentes, método y resultados del estudio.

\section{Investigaciones previas}

Nuestro análisis pretende observar algunos resultados de la transposición didáctica (Chevallard, 1991), esto es, los cambios del conocimiento matemático cuando es adaptado para la enseñanza. Desde el currículo pretendido al implementado en el aula, el currículo escrito y la forma en que lo interpretan los profesores, a través de los libros de texto, es una fase intermedia (Herbel, 2007). El libro de texto es un soporte curricular, a través del cual se vehicula el conocimiento que las instituciones educativas han de transmitir (Escolano, 2009). La presentación del tema y los problemas propuestos se basan con frecuencia en los presentados en ellos (Shield \& Dole, 2013). Cordero y Flores (2007) indican que el discurso matemático escolar es determinado con frecuencia por el libro de texto, que regula las acciones de enseñanza y aprendizaje, junto con las creencias de los profesores. Reys, Reys y Chavez (2004) sugieren que los libros de texto presentan las ideas matemáticas en diferentes contextos, a la vez que permiten a los estudiantes explorar diferentes ideas y facilitan el aprendizaje. Todo ello explica el interés que recibe la investigación sobre textos de matemáticas por la comunidad internacional (e.g., Fan \& Zhu, 2007).

La investigación específica sobre contenidos de estadística en los libros de texto de matemáticas es menor, aunque encontramos algunos ejemplos como los de Cobo y Batanero (2004) y Díaz-Levicoy (2014). Respecto a la correlación y regresión, destacamos las investigaciones de Sánchez Cobo (1999), quien estudia las definiciones de conceptos relacionados con la regresión en once libros de texto de Bachillerato, publicados entre 1977 y 1990, clasificándolas según se definan explícitamente o bien mediante una fórmula, o bien una mezcla de las anteriores.

Lavalle, Micheli y Rubio (2006) analizan la enseñanza de la correlación y regresión en siete textos argentinos dirigidos a estudiantes de Bachillerato, considerando los conceptos y procedimientos asociados, así como sus relaciones. Encuentran distintos niveles de profundidad en el tratamiento del tema. Solo un texto define el concepto de recta de regresión; cuatro incluyen la ecuación de la recta, siendo únicamente dos los que además utilizan funciones de ajuste distintas a la lineal. En la mayoría de los textos analizados por las autoras se trata también la estimación de la variable dependiente utilizando la función de ajuste. 
Por su parte, Gea et al. (2013) realizan un estudio empírico de algunas variables, como tipo e intensidad de la correlación en los problemas de correlación y regresión propuestos en una muestra de libros textos de Bachillerato de Ciencias Sociales. En Gea et al. (2014) analizan, en estos mismos libros de texto, el lenguaje utilizado, como por ejemplo, el lenguaje simbólico, expresiones algebraicas y verbales utilizadas, las representaciones gráfica y tabular de la distribución bidimensional, tema que el estudiante aprende antes de comenzar el estudio de la correlación y regresión. En Gea, Batanero, Fernandes y Gómez (2014) se estudia los conceptos ligados a la distribución de datos bidimensional tales como variable bidimensional, distribuciones unidimensionales asociadas a la distribución de datos bivariados, así como sus representaciones, entre otros. En este artículo se complementan los anteriores, estudiando los conflictos semióticos, tema que no ha sido considerado en investigaciones previas.

\section{Método}

Se analizaron dieciséis libros de textos de primer curso de Bachillerato, ocho de la especialidad en Humanidades y Ciencias Sociales y otros ocho de la especialidad en Ciencias y Tecnología. Todos los libros fueron publicados después de implantarse el currículo actual (MEC, 2007), y continúan siendo utilizados en los institutos de educación secundaria. Se eligieron, tras una consulta en la Consejería de Educación, por ser los más utilizados en la enseñanza pública en Andalucía y estar publicados en editoriales de gran tradición y prestigio en España. Se listan en el Anexo 1, donde hemos asignado un código a cada uno de estos libros, que será utilizado en las tablas de resultados. Al tratarse de una investigación cualitativa, la muestra utilizada es intencional; se trata de buscar una muestra que sea comprehensiva para capturar la mayor riqueza posible de la realidad analizada (Martínez, 2006).

Sobre estos textos se ha realizado un análisis de contenido, que asume que un texto puede dividirse en unidades que pueden clasificarse en un número reducido de categorías en función de variables subyacentes y que permiten realizar inferencias sobre su contenido (Noguero, 2002). El análisis adapta la metodología de Cobo y Batanero (2004): primeramente se identificaron en cada texto los capítulos correspondientes a la correlación y regresión; después, para cada capítulo se analizaron las situaciones problemáticas, lenguaje, conceptos, propiedades, procedimientos y argumentos.

Para cada uno de estos objetos se realiza un estudio detallado de su presentación, los ejemplos propuestos y el uso que se hace a lo largo del capítulo. La finalidad es identificar los posibles conflictos semióticos asociados, que se clasifican en categorías, siguiendo un proceso cíclico e inductivo, característico del analisis de contenido (Cook \& Reichardt, 2000). Seguidamente, se establece la presencia de cada una de las categorías en los libros de la muestra, a través de la comparación del contenido de estos textos, seleccionando ejemplos para facilitar su descripción y resumiendo los resultados en una tabla que facilita la obtención de conclusiones. Para asegurar la fiabilidad del proceso, se revisan los resultados del análisis con la ayuda de otros miembros del equipo de investigación.

A continuación se describen y analizan los conflictos semióticos identificados para cada uno de las tipos de objetos matemáticos considerados en nuestro estudio. 


\section{Resultados}

Como se ha indicado, cuando una persona realiza una actividad matemática, activa su propia trama de funciones semióticas, que serán correctas si se conforman a la institución; en otro caso, se considera que existe un conflicto semiótico (Godino, 2002; Godino et al., 2006). Los autores de los libros de texto pueden reflejar dichos conflictos de un modo explícito o implícito en el tema; dichos conflictos podrían posteriormente transmitirse a los estudiantes y explicar algunas de sus dificultades y limitaciones en los aprendizajes.

Consideraremos que existe dicho conflicto de manera explícita cuando identificamos una asignación de significado a un objeto matemático que no concuerda con la correcta institucionalmente; será implícito si la asignación no llega a ser incorrecta, aunque sí imprecisa o incompleta, ya que puede originar un conflicto en el estudiante, que al apoyarse en el libro de texto puede conducirle a realizar generalizaciones en forma excesivamente amplia, o interpretaciones inadecuadas.

\subsection{Conflictos semióticos potenciales ligados al lenguaje}

Representación gráfica incorrecta de una distribución bivariante. Hemos considerado que se presenta este conflicto cuando se utilizan gráficos estadísticos no adecuados al tipo de datos que se representa. Esto ocurre en los textos que intercambian histogramas con diagramas de barras tridimensionales, es decir, no tienen en cuenta que estas representaciones se deben utilizar dependiendo de si las variables son continuas o categóricas. Por ejemplo, hemos encontrado en los textos [H1] y [T1] la representación gráfica de una variable discreta (con valores enteros) a partir de su representación tabular mediante un histograma, en lugar de un diagrama de barras (Figura 1). Ello puede llevar al estudiante a confundir estos dos tipos de gráficos o a no diferenciar los casos en que uno y otro debe aplicarse. Esta situación se repite en [H7] y [T7]. Se trataría de un conflicto explícito pues los autores utilizan una representación de la distribución no acorde con las correctas desde el punto de vista de la institución matemática.

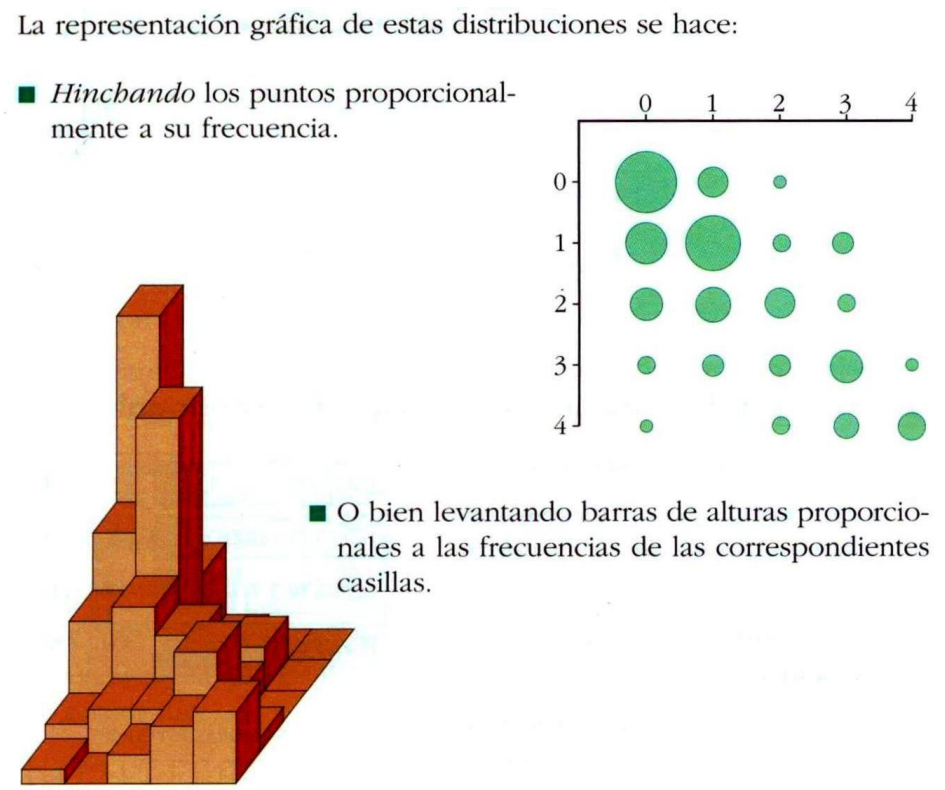

Figura 1. Representación gráfica de una distribución bidimensional ([H1], p. 233). 
Hemos encontrado también los siguientes usos imprecisos del lenguaje matemático (todos ellos implican conflictos semióticos implícitos):

Identificación de un concepto con su representación tabular y/o gráfica. El carácter no ostensivo de los objetos matemáticos, hace necesario el uso de representaciones de los mismos para su trabajo en el aula. Duval (1993) indica el interés didáctico de manejar diferentes representaciones, pero también recuerda que los objetos matemáticos nunca deben ser confundidos con su representación. En nuestro estudio, hemos encontrado algunos libros que inducen esta confusión, pues no separan claramente la representación del objeto (expresión) del objeto mismo (contenido) al establecer una relación entre los mismos (función semiótica). Así, los textos [H1] y [T1] definen inicialmente la distribución bidimensional mediante un ejemplo de representación gráfica y otra tabular; no es hasta más adelante cuando se da una definición más precisa del concepto: "El conjunto de pares de valores $\left(x_{1}, y_{1}\right)$, $\left(x_{2}, y_{2}\right) \ldots\left(x_{n}, y_{n}\right)$ se llama una distribución bidimensional” ([T1], p.333). Por otro lado, en estos mismos textos, la distribución marginal se introduce únicamente nombrándola mediante una etiqueta de una tabla de frecuencias ([H1], p.237) y no llega a definirse, a pesar de su relevancia. Este hecho puede provocar que el alumno no llegue a comprender este concepto o lo interprete simplemente como sinónimo de "total" de una fila o columna y no como conjunto de valores de la variable fila o columna, junto con las correspondientes frecuencias marginales.

Lenguaje simbólico inapropiado o impreciso. Hemos encontrado ejemplos en los que el lenguaje simbólico podría inducir conflicto en los estudiantes. Por ejemplo, en [H4] y [T4], al definir el concepto de frecuencia marginal, se utilizan tablas en las que aparecen estas frecuencias con una notación no habitual, como en el ejemplo que reproducimos en la Tabla 1 . Los símbolos $A_{i}$ y $B_{j}$ se refieren a las frecuencias absolutas marginales de las variables $X_{i}$ e $Y_{j}$ (para $i=1, \ldots, \mathrm{n}$ y $\left.j=1, \ldots, \mathrm{m}\right)$, respectivamente. El uso de este lenguaje podría generar un conflicto semiótico en la interpretación de la notación, pudiendo el estudiante entender las expresiones $\left(f_{r}\right) A_{n}$ ó $\left(f_{r}\right) B_{m}$ como producto de dos elementos.

Tabla 1. Tabla de doble entrada de frecuencias relativas ([H4], p. 219).

\begin{tabular}{ccccccc}
\hline & $\mathbf{y}_{\mathbf{1}}$ & $\mathbf{y}_{\mathbf{2}}$ & $\mathbf{y}_{\mathbf{3}}$ & $\cdots$ & $\mathbf{y}_{\mathbf{m}}$ & $\sum_{j=1}^{m}\left(f_{r}\right)_{i j}$ \\
\hline $\mathbf{x}_{\mathbf{1}}$ & $\left(\mathrm{f}_{\mathrm{r}}\right)_{11}$ & $\left(\mathrm{f}_{\mathrm{r}}\right)_{12}$ & $\left(\mathrm{f}_{\mathrm{r}}\right)_{13}$ & & $\left(\mathrm{f}_{\mathrm{r}}\right)_{1 \mathrm{~m}}$ & $\left(\mathrm{f}_{\mathrm{r}}\right) \mathrm{A}_{1}$ \\
$\mathbf{x}_{\mathbf{2}}$ & $\left(\mathrm{f}_{\mathrm{r}}\right)_{21}$ & $\left(\mathrm{f}_{\mathrm{r}}\right)_{22}$ & $\left(\mathrm{f}_{\mathrm{r}}\right)_{23}$ & & $\left(\mathrm{f}_{\mathrm{r}}\right)_{2 \mathrm{~m}}$ & $\left(\mathrm{f}_{\mathrm{r}}\right) \mathrm{A}_{2}$ \\
$\mathbf{x}_{\mathbf{3}}$ & $\left(\mathrm{f}_{\mathrm{r}}\right)_{31}$ & $\left(\mathrm{f}_{\mathrm{r}}\right)_{32}$ & $\left(\mathrm{f}_{\mathrm{r}}\right)_{33}$ & & $\left(\mathrm{f}_{\mathrm{r}}\right)_{3 \mathrm{~m}}$ & $\left(\mathrm{f}_{\mathrm{r}}\right) \mathrm{A}_{3}$ \\
$\cdots$ & $\left(\mathrm{f}_{\mathrm{r}}\right)_{\mathrm{n} 1}$ & $\left(\mathrm{f}_{\mathrm{r}}\right)_{\mathrm{n} 2}$ & $\left(\mathrm{f}_{\mathrm{r}}\right)_{\mathrm{n} 3}$ & & $\left(\mathrm{f}_{\mathrm{r}}\right)_{\mathrm{nm}}$ & $\left(\mathrm{f}_{\mathrm{r}}\right) \mathrm{A}_{\mathrm{n}}$ \\
\hline $\mathbf{x}_{\mathbf{n}}$ & $\left(\mathrm{f}_{\mathrm{r}}\right)_{\mathrm{B}_{1}}$ & $\left(\mathrm{f}_{\mathrm{r}}\right)_{\mathrm{B}_{2}}$ & $\left(\mathrm{f}_{\mathrm{r}}\right)_{\mathrm{B}_{3}}$ & $\cdots$ & $\left(\mathrm{f}_{\mathrm{r}}\right)_{\mathrm{B}_{\mathrm{m}}}$ & 1 \\
\hline$\sum_{i=1}^{n}\left(f_{r}\right)_{i j}$ & & & & & \\
\hline
\end{tabular}

Por otra parte, en [H1], [H5], [H6], [T1], [T5] y [T6] no quedan lo suficientemente claros los símbolos utilizados al introducir la fórmula de la covarianza, como se muestra en la Figura 2. Es particularmente confuso el ejemplo que se presenta, que muestra los datos en una tabla de doble entrada, donde sería necesario utilizar dos índices $i, j$ dado que los datos poseen frecuencia distinta a la unidad. Sin embargo, en 
la fórmula se utiliza únicamente el índice $i$. [H2], [H8] y [T2] previenen este conflicto implícitamente, cuando se indica al alumno la utilidad de transformar la tabla de doble entrada en una tabla simple o listado de datos para trabajar con ellos más fácilmente. Finalmente, en [H5] y [T5] el uso del lenguaje podría dificultar al estudiante la conexión entre representación tabular y gráfica de una distribución bidimensional, pues se presenta ambas representaciones con una notación diferente en un mismo ejemplo (Figura 3).
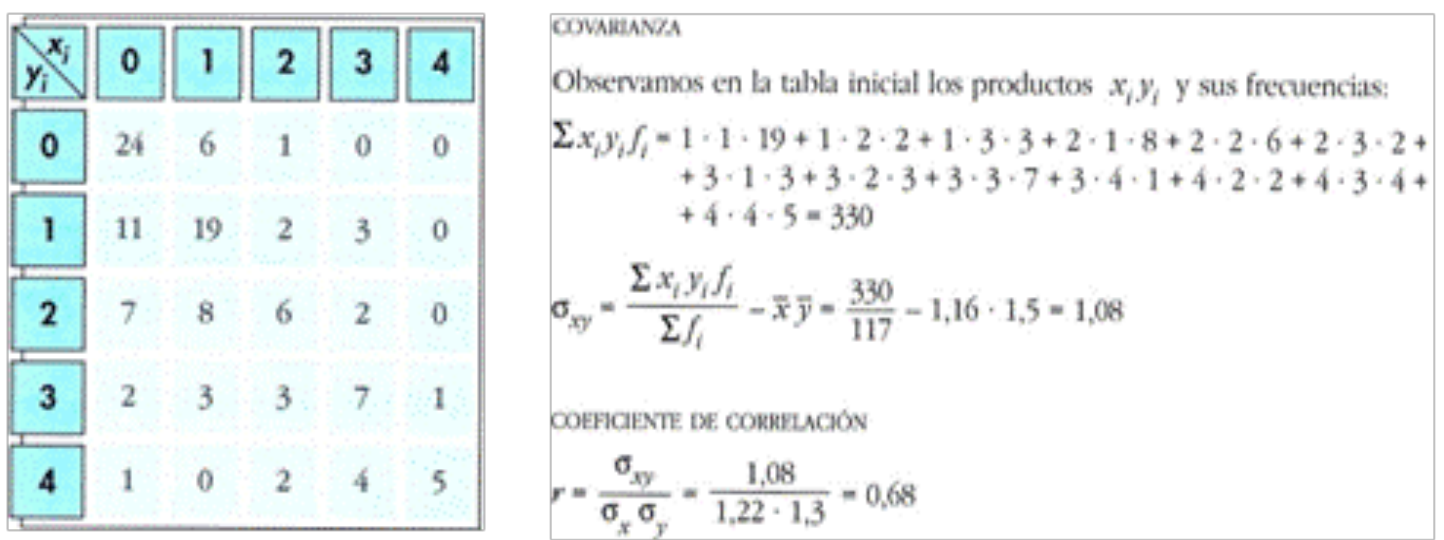

Figura 2. Cálculo de la covarianza con notación imprecisa ([H1], p. 237).
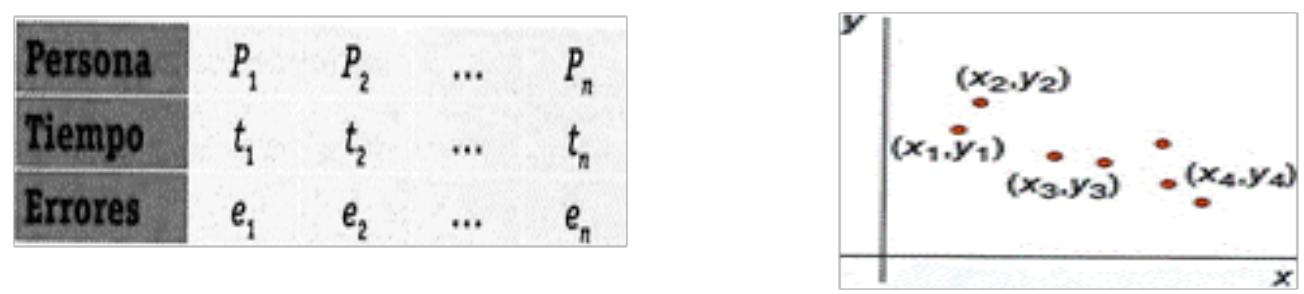

Figura 3. Proceso de traducción entre la representación gráfica y tabular con diferente notación ([H5], p. 250).

\subsection{Conflictos semióticos potenciales ligados a la definición de conceptos}

Independencia y correlación nula. Encontramos textos como [H1], [H2], [H8], [T1], [T2] y [T8], que tratan como equivalentes los términos independencia y correlación nula (variables incorreladas), cuando en todos los casos se refieren a la noción de independencia. Estos términos no son equivalente, pues, por ejemplo, si la relación entre las variables es parabólica, se puede obtener un coeficiente de correlación nulo (serían incorreladas) con dependencia incluso funcional. Al identificar conceptos no equivalentes se produce un conflicto semiótico, que en este caso, es explícito.

Por ejemplo, en [H8] y [T8] se define de manera incorrecta la independencia entre variables: "La correlación es nula cuando no existe ninguna relación entre ambas variables: en este caso, los puntos del diagrama están esparcidos al azar, sin tender a formar ninguna línea, y se dice que las variables están incorreladas." ([H8], p. 252). Esta definición se acompaña de varios diagramas de dispersión para que el estudiante distinga el tipo de dependencia según intensidad, signo y tipo de función (lineal o curvilínea); más adelante se muestra un ejemplo con variables claramente independientes (Figura 4). 


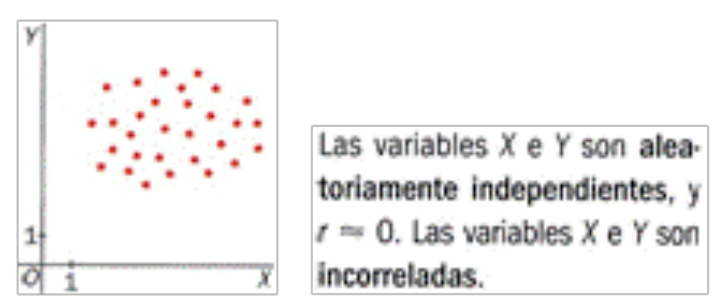

Figura 4. Introducción de la independencia en varios momentos ([H8], pp. 252 y 253).

Dependencia y correlación. Algunos textos analizados identifican explícitamente correlación con relación estadística/aleatoria entre las variables, aunque no son equivalentes, pues el término correlación sólo se aplica a variables numéricas, mientras que la dependencia entre variables cualitativas se denomina asociación. Por ello, un uso generalizado del término correlación podría producir un conflicto semiótico en el estudio posterior de la asociación con variables cualitativas. Por ejemplo, en [H6] y [T6] se define la dependencia estadística del siguiente modo: "La relación entre dos variables también puede ser estadística, y recibe entonces el nombre de correlación." ([H6], p. 180), definiendo más adelante la correlación como: "La correlación es la relación que existe entre dos variables estadísticas unidimensionales que constituyen una variable estadística bidimensional". En [H8] y [T8] se indica: "Se llama correlación a la "relación o dependencia" que existe entre las dos variables que intervienen en una distribución bidimensional" ([H8], p.252). Esta imprecisión se repite al hablar de la intensidad de la dependencia, como ocurre en [H5] y [T5], donde se señala: "La correlación puede calificarse como fuerte cuando el grado de dependencia es alto; y como débil en caso contrario" ([T5], p.358).

Otro conflicto relacionado es identificar dependencia funcional con correlación no lineal, como se observa en la Figura 5, donde se muestra un diagrama de dispersión con una dependencia claramente aleatoria, pero que se describe como dependencia funcional por el hecho de no tratarse de una dependencia lineal.

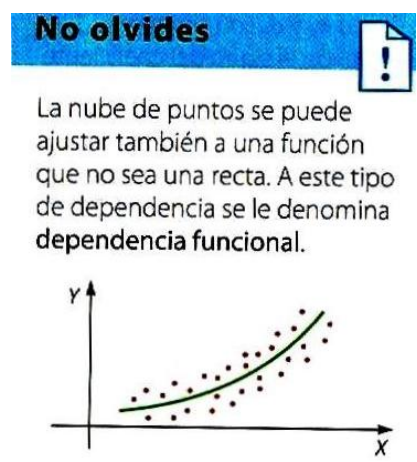

Figura 5. Definición de dependencia funcional ([H7], p. 247).

Por otro lado, [H2] y [T2] usan el término "correlación funcional" para referirse a la dependencia funcional, llegando a enunciar la siguiente propiedad ([H2], p. 249): "Si $r=-1$ or $r=1$, la correlación es perfecta o funcional".

Igualmente advertimos imprecisiones, relacionadas con las definiciones de conceptos, que suponen conflictos implícitos y describimos a continuación:

Variable estadistica bidimensional. Una imprecisión en su definición es la que presentan los textos que reducen el significado del concepto cuando limitan su 
definición a variables estadísticas unidimensionales cuantitativas, como ocurre en [H8] y [T8]: "Una variable estadística bidimensional $(X, Y)$ es el resultado del estudio de dos características cuantitativas $X$ e $Y$ en los individuos de una población." ([H8], p.248).

Dependencia funcional. Algunos textos identifican la dependencia funcional con la existencia de una gráfica dada por una expresión matemática a la cual se ajusta perfectamente el diagrama de dispersión. Esta es una definición limitada pues hay funciones para las que no encontramos expresión funcional exacta; por ejemplo, las mismas funciones de distribución en estadística; por tanto puede llevar a un conflicto en la comprensión de la idea de función en los estudiantes. Así, el criterio "Los puntos de la nube se sitúan sobre una curva cuya expresión matemática podríamos determinar" ([H3], p.222) podría inducir la concepción algebraica de la asociación (en este caso, para la dependencia funcional) descrita por Estepa (1994).

Coeficiente de correlación lineal. Encontramos textos ([H1], [H2], [T1] y [T2]) donde no se indica con claridad que el coeficiente de correlación lineal únicamente mide la relación de tipo lineal. Esta imprecisión puede inducir un conflicto posterior en el estudio de la correlación curvilínea, haciendo suponer que el coeficiente de correlación lineal pudiera informar de la intensidad este otro tipo de dependencia. Un ejemplo lo encontramos en [H5] y [T5], donde se indica:

Hasta ahora nos hemos limitado, y no es poco, a decir cuándo dos variables están correlacionadas. Primero, representábamos el diagrama de dispersión y decíamos que, cuando una recta se ajustaba bien a la nube de puntos, entonces la correlación era fuerte. Después, con el coeficiente $r$, dábamos una medida del sentido y fuerza de la correlación ([T5], p. 364).

El caso más destacado de esta imprecisión aparece en los textos [H2] y [T2], con la siguiente proposición, donde se supone que el coeficiente de correlación $r$ mide la distancia al centro de gravedad (propiedad que no es cierta):

El coeficiente de correlación de Pearson indica la correlación que existe entre las dos variables; es decir, si los puntos están muy próximos o alejados del centro de gravedad. ([H2], p. 249).

Correlación y regresión. Encontramos varios textos en los que el concepto de correlación se confunde, en ocasiones, con el de regresión. Por ejemplo, [H4] define la correlación como: "la dependencia que existe entre las dos variables de una distribución bidimensional” ([H4], p. 224), y más adelante, al tratar sus características, se incluye como cualidad de la correlación el tipo de función de ajuste (que correspondería a la regresión) del siguiente modo:

Se pueden distinguir los siguientes casos:

- La correlación es de tipo funcional si existe una función tal que la satisfacen todos los valores de la distribución.

- La correlación es lineal o curvilínea cuando la nube de puntos se agrupa en torno a una línea recta o a una curva, respectivamente. [...] ([H4], p.224).

Incluso en algunos textos donde encontramos una definición clara del concepto de regresión, como [H5], el sentido dado a la correlación incluye al de regresión. Destacamos también [H7] y [T7], que no incluyen otra definición de regresión que la definición de las rectas de ajuste mínimo cuadráticas, pero plantea la tarea que se muestra en la Figura 6, donde es claro que la segunda figura no es susceptible de ser modelizada con una recta. 
38 Representa, sin hallar su ecuación, la recta de regresión ๑०० correspondiente a estas variables.
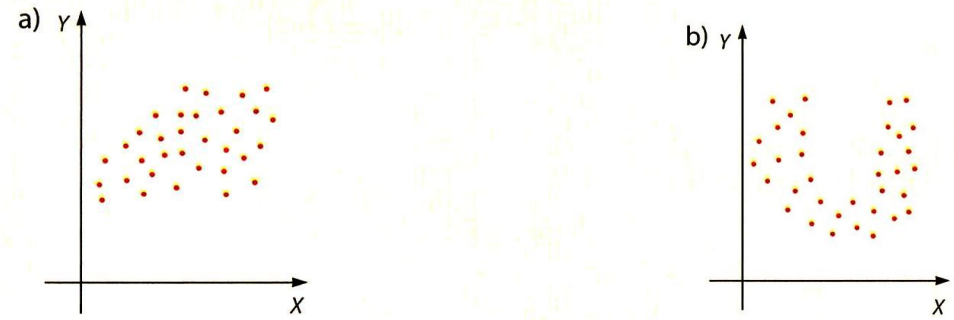

Figura 6. Tarea sobre regresión lineal ([H7], p. 260).

\subsection{Conflictos semióticos potenciales ligados a las proposiciones}

En ocasiones, se atribuyen a los conceptos propiedades que no poseen, o bien se realiza una generalización muy amplia de otras, lo que podría influir en la posterior adquisición, por parte de los estudiantes de conflictos o concepciones erróneas sobre la correlación como la unidireccional o causal (Estepa, 1994; Estepa \& Batanero, 1995). Algunas de ellas son explícitas:

Interpretación de resúmenes estadísticos en un análisis unidimensional. En algunos ejercicios, como el que se muestra en la Figura 7, se interpretan incorrectamente algunos estadísticos univariantes. En este caso se considera el coeficiente de variación como un coeficiente de concentración. Por otro lado, se indica que al ser el valor de la desviación típica grande (de hecho no es mucho mayor que la media), la muestra no es representativa. Pero la representatividad de la muestra no tiene nada que ver con su dispersión, sino con el hecho de que los valores centrales sean próximos a los de la población, y que la muestra tenga tamaño suficiente, así como dispersión y forma similar a la población.

Estas son las medidas estadísticas de un estudio sobre el número de roturas que sufrieron unos alfileres a los que se les sometió a una prueba.

$$
\begin{aligned}
& \bar{x}=0,7 \quad M e=0 \quad M o=0 \\
& \sigma=0,96 \quad C V=1,37
\end{aligned}
$$

Interpreta estas medidas estadísticas

PRIMERO. Se compara $\bar{x}$ con la mediana y la moda. La media, $\bar{x}=0,7$, indica que el número medio de roturas es casi 1 . Sin embargo, la mayoría de los alfileres no ha sufrido roturas $(M o=0)$, y lo mismo indica la mediana $(M e=0)$, es decir, más de la mitad de los alfileres no ha sufrido ninguna rotura durante el estudio.

SEGUNDO. Se estudia el valor del coeficiente de variación, $\mathrm{CV}=1,37$ es un valor muy grande, por lo que los datos no están demasiado concentrados

TERCERO. Se estudia el valor de la desviación típica y se compara con el valor de la media. El valor de la desviación típica es mayor que el de la media. Esto explica por qué mientras la mediana y la moda indican que el mayor número de alfileres no ha tenido roturas, la media de roturas ha sido casi de 1. Al tener una desviación típica tan grande, la media no es muy representativa.

Figura 7. Tarea de interpretación de estadísticos en variable unidimensional ([T7], p.312).

En otro caso, encontramos propiedades incorrectas que se atribuyen de manera implícita, como las siguientes. 
Sentido de la correlación. Algunos textos asumen correlación entre las variables únicamente cuando el aumento de una de ellas implique el aumento de la otra, llegando a considerar independencia en cualquier otro caso. Esta descripción podría incidir en la concepción unidireccional sobre la correlación (Estepa, 1994). Ninguno de los textos de la muestra advierten de la importancia de observar el decrecimiento conjunto de las variables en el estudio de correlación. En algunos textos, como [H2] y [T2], se advierte de la importancia que tiene la posición de los datos (cuatro cuadrantes en que el plano se divide mediante el trazado de las dos medias de las variables $X$ e $Y$ ) para valorar el signo de la covarianza y la correlación. Aún así, las proposiciones que presentan invitan a confusión ([H2], p. 246):

Según sea el signo de la covarianza, se interpreta:

a. Covarianza positiva: al aumentar los valores de la variable $X$, aumentan los valores de la variable $Y$. La nube de puntos se orienta a la derecha y hacia arriba.

b. Covarianza negativa: al aumentar los valores de la variable $\mathrm{X}$, disminuyen los valores de la variable Y. La nube de puntos se orienta a la derecha y hacia abajo.

Correlación y causalidad. Algunos textos como [T5] indican, al estudiar la bondad de ajuste, que la varianza de la variable dependiente que no es explicada por la variable independiente será debida a otras causas, como muestra el siguiente texto:

Por tanto, el coeficiente de determinación $\mathrm{r}^{2}=0,94^{2}=0,88$. Esto significa que en los niños de nuestro ejemplo, el $88 \%$ de la altura se explica por la edad; el resto, hasta el $100 \%$, será debido a otras causas; altura de sus padres, dieta,... ([T5], p. 363).

Este tipo de explicación podría inducir la concepción causal, que consiste en considerar asociación entre variables estadísticas solamente cuando exista causa y efecto entre ellas.

\subsection{Conflictos semióticos potenciales ligados a los procedimientos}

En general, se deben a descripciones confusas o imprecisas de los procedimientos, por lo cual se trataría de conflictos implícitos o potenciales. Hemos encontrado los siguientes:

Descripción confusa del cálculo de la covarianza o correlación. Cuando se introduce la covarianza, sería recomendable que la fórmula de cálculo venga acompañada de todas las expresiones equivalentes que se pretendan utilizar en el texto, como hace [H3]. Sin embargo, algunos textos introducen el procedimiento sin explicar los pasos o bien usan notación sumatoria donde no se indica el rango de variación de la suma. Un ejemplo es [H1], que no especifica la variación de los índices del sumatorio en la fórmula de la covarianza; por tanto, no indica qué valores hay que sumar, potenciando un conflicto en el alumno en cuanto a la aplicación de esta fórmula (véase Figura 2).

Explicación confusa de la construcción de tablas bidimensionales. En algunos textos, por ejemplo [H7] y [T7], se incluye una explicación completa de la construcción de tablas bidimensionales con datos agrupados (Figura 8). Sin embargo, en la descripción observamos varios aspectos confusos. Por un lado, se presentan los datos inicialmente en forma de listado, y el alumno ha de construir, sin indicaciones, la variable estadística bidimensional. Por otro lado, se le deja clasificar los puntos obtenidos en intervalos bidimensionales, presentando la tabla final como listado, sin dar la posibilidad de que aparezcan otras combinaciones de las dos variables; por ejemplo, un estudiante con puntuación $(95,3)$. Otra complicación se produce al sustituir los intervalos por su marca de clase en la tabla final. Esta combinación de 
imprecisiones supone una gran dificultad de comprensión del método para el alumno, que le puede llevar a conflictos en la construcción de la tabla, la interpretación de los intervalos y sus extremos o incluso la identificación correcta de la distribución bidimensional.

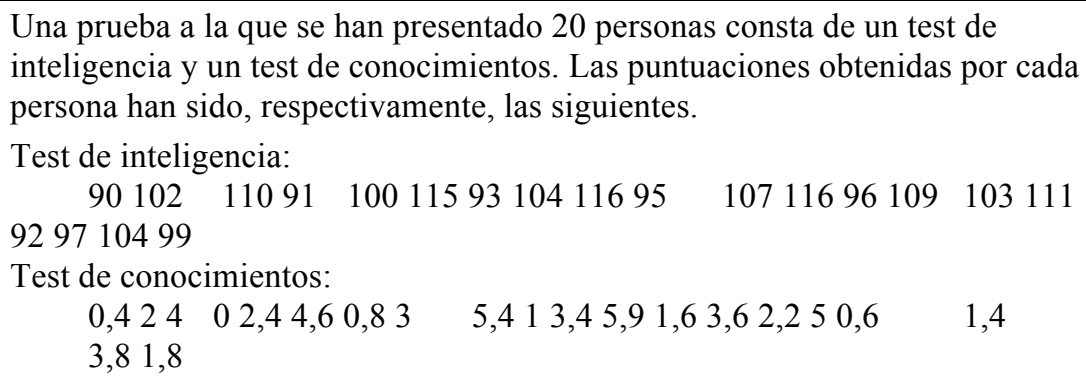

Agrupa las puntuaciones del test de inteligencia en intervalos de 10 puntos, y las puntuaciones del test de conocimientos, en intervalos de 2 puntos. Construye la tabla de frecuencias simple para datos agrupados.

PRIMERO. Se determinan los intervalos para la primera variable y las marcas de clase correspondientes. Como las puntuaciones del test de inteligencia van de 90 a 116 puntos, se puede hacer la división en tres intervalos:

$$
[90,100) \quad[100,110) \quad[110,120)
$$

Las marcas de clase son los puntos medios de cada intervalo, esto es:

$$
95 \quad 105 \quad 115
$$

SEGUNDO. Se determinan los intervalos para la segunda variable y las marcas de clase correspondientes. Como las puntuaciones del test de conocimientos van de 0 a 5,9 puntos, podemos hacer la división en tres intervalos:
$[0,2)$

En este caso, las marcas de clase son:

$$
1 \quad 3
$$

TERCERO. Se construye la tabla de frecuencias simple para datos agrupados. Para ello se cuentan los datos que pertenecen a cada intervalo, para determinar las frecuencias absolutas, y se representa cada intervalo mediante su marca de clase

\begin{tabular}{|l|c|c|c|}
\hline Puntuaciones del test de inteligencia & 95 & 105 & 115 \\
\hline Puntuaciones del test de conocimientos & 1 & 3 & 5 \\
\hline Frecuencias absolutas & 8 & 7 & 5 \\
\hline
\end{tabular}

Figura 8. Ejercicio resuelto de representación tabular de datos bidimensionales ([H7], p. 254).

Explicación confusa de representaciones gráficas de datos bidimensionales. Es difícil que un estudiante aprenda a construir adecuadamente un diagrama de barras, con las descripciones que aparecen en textos como [H2] y [T2], donde tan sólo se muestra un ejemplo de este gráfico (Figura 9). La construcción no viene acompañada de alguna aclaración en cuanto a la situación de las barras. Además, no se indica que el gráfico no es adecuado para representar una variable estadística bidimensional continua, donde habría que utilizar un histograma. Tampoco se precisa aspectos de la proporcionalidad de la altura de las barras y la frecuencia de los datos (ver Figura 1). 


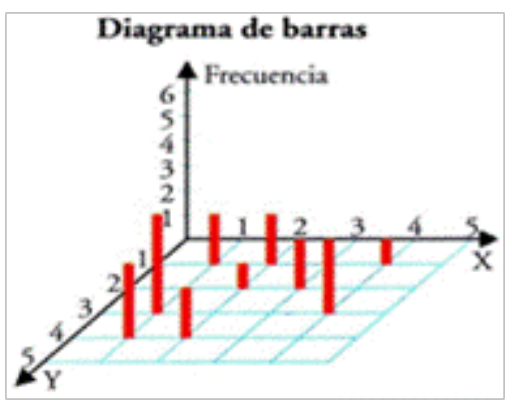

Figura 9. Representación gráfica de datos bidimensionales ([T2], p. 317).

Procedimientos confusos en el análisis de regresión. Algunos textos no diferencian, en el cálculo de las rectas de regresión, la variable dependiente e independiente (con el posible riesgo para la estimación). La confusión entre variable dependiente e independiente ha sido encontrada en algunas investigaciones previas como la de Ruiz (2013). Pensamos que si la diferencia no está clara en los textos, se puede potenciar este conflicto. Destacamos un ejercicio en el texto [H1], donde se estima un valor de $y$ mediante la recta de regresión de $Y$ sobre $X$, y un valor de $x$ con la inversa de dicha recta; lo que sería un procedimiento incorrecto. Es cierto que se lleva a cabo bajo una correlación casi perfecta $(r=0$ '9994), y que se advierte que las estimaciones de la variable independiente se suelen realizar sobre "la otra recta de regresión" ([H1], p. 231), pero pensamos que el alumno podría generalizar en forma incorrecta este procedimiento.

\subsection{Conflictos semióticos potenciales ligados a los argumentos}

Finalmente, se ha observado que algunos textos presentan argumentos que implícitamente podrían inducir conflictos semióticos en los estudiantes. Un ejemplo es el siguiente: "Si los puntos siguen, aunque sea aproximadamente, una configuración rectilínea, diremos que hay dependencia entre ellos" ([H7], p.247). Aunque el texto añade descripciones y ejemplos en que se aprecia el signo e intensidad de la dependencia lineal, la descripción citada es incorrecta o cuanto menos confusa, pues implícitamente supone una concepción funcional de la dependencia (Estepa. 1994). En la modalidad científico-tecnológica ([T7]) este argumento aparece pero utilizando el término dependencia lineal en lugar de dependencia, lo que sería correcto.

\subsection{Síntesis de conflictos semióticos observados}

Como resumen de los conflictos semióticos potenciales observados, presentamos las Tablas 2 y 3 , donde se aprecia que todos los textos incluyen algún tipo de conflicto, a menos en forma implícita, debido a imprecisión en la presentación de los diferentes objetos matemáticos.

No se aprecian grandes diferencias en los textos de cada modalidad, salvo [T5] y [T7], donde se trata la correlación y causalidad o la interpretación de estadísticos, respectivamente, con presencia de conflictos potenciales. En estos casos, la editorial no incluye la enseñanza de estas proposiciones en el Bachillerato de Humanidades y Ciencias Sociales, en el primer caso, porque el coeficiente de determinación no se define en [H5], y en el segundo, pues no se incluye el análisis unidimensional de modo tan extenso. Observamos variabilidad en los libros ya que algunos presentan apenas 
unos pocos conflictos, mientras que en otros aparece un gran número de ellos.

Tabla 2. Conflictos semióticos en los textos analizados de Humanidades y Ciencias Sociales.

\begin{tabular}{|c|c|c|c|c|c|c|c|c|c|}
\hline & & H1 & H2 & H3 & H4 & H5 & H6 & H7 & H8 \\
\hline & Ligados al lenguaje & & & & & & & & \\
\hline Explícito & Representación incorrecta & $\mathrm{x}$ & & & & & & $\mathrm{x}$ & \\
\hline \multirow[t]{2}{*}{ Implícitos } & Confusión objeto-representación & $\mathrm{x}$ & & & & & & & \\
\hline & Lenguaje impreciso & $\mathrm{x}$ & & & $\mathrm{x}$ & $\mathrm{x}$ & $\mathrm{x}$ & & \\
\hline \multirow{3}{*}{ Explícitos } & Ligados a los conceptos & & & & & & & & \\
\hline & Dependencia y correlación & & $\mathrm{x}$ & & & & $\mathrm{x}$ & $\mathrm{x}$ & $\mathrm{x}$ \\
\hline & Independencia y correlación & $\mathrm{x}$ & $\mathrm{x}$ & & & $\mathrm{x}$ & & & $\mathrm{x}$ \\
\hline \multirow[t]{4}{*}{ Implícitos } & Variable bidimensional & & & & & & & & $\mathrm{x}$ \\
\hline & Dependencia funcional & & & $\mathrm{x}$ & & $\mathrm{x}$ & & & \\
\hline & Correlación y coeficiente de correlación & $\mathrm{x}$ & $\mathrm{x}$ & & & $\mathrm{x}$ & & & \\
\hline & Correlación y regresión & & & $\mathrm{x}$ & $\mathrm{x}$ & $\mathrm{x}$ & $\mathrm{x}$ & $\mathrm{x}$ & \\
\hline & Ligados a las proposiciones & & & & & & & & \\
\hline Explícito & Interpretación de estadísticos & & & & & & & & \\
\hline \multirow[t]{2}{*}{ Implícitos } & Sentido de la correlación & & $\mathrm{x}$ & & & & & & \\
\hline & Correlación y causalidad & & & & & & & & \\
\hline \multirow{6}{*}{ Implícitos } & Ligados a los procedimientos & & & & & & & & \\
\hline & Fórmulas de cálculo & $\mathrm{x}$ & & & & & & & \\
\hline & Construcción de tablas & & & & & & & $\mathrm{x}$ & \\
\hline & Construcción de gráficas & & $x$ & & & & & & \\
\hline & Procedimientos confusos en regresión & $\mathrm{x}$ & & & & & & & $\mathrm{x}$ \\
\hline & Ligados a los argumentos & & & & & & & $\mathrm{x}$ & $\mathrm{x}$ \\
\hline
\end{tabular}

Tabla 3. Conflictos semióticos en los textos analizados de Ciencias y Tecnología.

\begin{tabular}{|c|c|c|c|c|c|c|c|c|c|}
\hline & & T1 & $\mathbf{T 2}$ & T3 & T4 & T5 & T6 & $\mathbf{T 7}$ & T8 \\
\hline & Ligados al lenguaje & & & & & & & & \\
\hline Explícito & Representación inadecuada & $x$ & & & & & & $\mathrm{x}$ & \\
\hline \multirow[t]{2}{*}{ Implícitos } & Confusión objeto-representación & $\mathrm{x}$ & & & & & & & \\
\hline & Lenguaje impreciso & $\mathrm{x}$ & & & $\mathrm{x}$ & $\mathrm{x}$ & $\mathrm{x}$ & & \\
\hline & Ligados a los conceptos & & & & & & & & \\
\hline \multirow[t]{2}{*}{ Explícitos } & Dependencia y correlación & & $\mathrm{x}$ & & & & $x$ & $\mathrm{x}$ & $\mathrm{x}$ \\
\hline & Independencia y correlación & $\mathrm{x}$ & $\mathrm{x}$ & & & $\mathrm{x}$ & & & $\mathrm{x}$ \\
\hline \multirow[t]{4}{*}{ Implícitos } & Variable bidimensional & & & & & & & & $\mathrm{x}$ \\
\hline & Dependencia funcional & & & $\mathrm{x}$ & & $\mathrm{x}$ & & & \\
\hline & Correlación y coeficiente de correlación & $\mathrm{x}$ & $\mathrm{x}$ & & & $\mathrm{x}$ & & & \\
\hline & Correlación y regresión & & & $\mathrm{x}$ & $\mathrm{x}$ & $\mathrm{x}$ & $\mathrm{x}$ & $\mathrm{x}$ & \\
\hline & Ligados a las proposiciones & & & & & & & & \\
\hline Explícito & Interpretación de estadísticos & & & & & & & $\mathrm{x}$ & \\
\hline \multirow[t]{3}{*}{ Implícitos } & Sentido de la correlación & & $\mathrm{x}$ & & & & & & \\
\hline & Correlación y causalidad & & & & & $x$ & & & \\
\hline & Ligados a los procedimientos & & & & & & & & \\
\hline \multirow[t]{5}{*}{ Implícitos } & Fórmulas de cálculo & $\mathrm{x}$ & & & & & & & \\
\hline & Construcción de tablas & & & & & & & $\mathrm{x}$ & \\
\hline & Construcción de gráficas & & $x$ & & & & & & \\
\hline & Procedimientos confusos en regresión & $\mathrm{x}$ & & & & & & & $\mathrm{x}$ \\
\hline & Ligados a los argumentos & & & & & & & $\mathrm{x}$ & $\mathrm{x}$ \\
\hline
\end{tabular}

Donde más conflictos potenciales aparecen es en la introducción de los conceptos. Al tratar de ponerlos al alcance de los estudiantes de este nivel, la transposición didáctica ocasiona cambio del significado de dichos conceptos. Es importante la 
vigilancia epistemológica para no llegar a fenómenos de deslizamiento de significado.

El lenguaje matemático se presenta también con poco rigor. Respecto a los procedimientos y argumentos no encontramos tantos conflictos.

\section{Conclusiones}

Nuestro análisis dio como resultado la identificación de algunos aspectos en que la descripción de los diferentes objetos matemáticos podría llevar a ocasionar conflictos semióticos en los estudiantes, debido a la introducción, uso o interpretación inadecuada de definiciones o representaciones de dichos objetos (funciones semióticas que no concuerdan plenamente con el significado institucional).

Se describieron y clasificaron estos conflictos potenciales dependiendo de si se referían al lenguaje, conceptos, propiedades, procedimientos o argumentos. Para cada uno de ellos se analizan las razones por las cuáles consideramos que se establecen funciones semióticas inadecuadas al describir un tipo de objeto, y se sugieren formas en que el profesor puede evitar dichos conflictos en los estudiantes.

Como hemos indicado anteriormente, se encontraron una gran variedad de conflictos semióticos potenciales, al igual que en otros estudios previos de libros de texto respecto a otros conceptos matemáticos (por ejemplo en Mayén, 2009). Todos estos conflictos se han analizado, ejemplificado y clasificado en el estudio.

Como consecuencia, observamos que la presentación de algunas propiedades (o la ausencia de dicha presentación) podría inducir conflictos semióticos en los estudiantes, como ocurre con el sentido de la correlación. Se suele describir, generalmente de manera implícita, que el sentido de la dependencia proviene de un aumento conjunto en las variables analizadas, pero esta variación también lleva asociado un decrecimiento conjunto de las mismas. Esta propiedad debiera ser trabajada por los estudiantes junto al profesor, ya que pudiera potenciar una concepción unidireccional en el estudiante (Estepa \& Batanero, 1995). En menor medida encontramos textos que incidan en la concepción causal, llegando a ser una de las concepciones más resistentes al cambio en los estudiantes (Estepa, 1994).

La mayoría de conflictos semióticos que encontramos se asocian al tratamiento del lenguaje y a los conceptos implicados en el tema. En cuanto al lenguaje destacamos, entre otros, confundir un objeto con su representación gráfica, confusión entre diagrama de barras e histograma, o el diagrama de dispersión y el gráfico de burbujas. Las imprecisiones en el lenguaje, así como de los procedimientos asociados a estos objetos, puede potenciar errores en los estudiantes y sus interpretaciones sobre la correlación en los mismos (Estepa, 2008). Otros ejemplos son el uso de simbolizaciones inadecuadas o imprecisas.

Encontramos una generalización demasiado amplia del concepto de correlación o la equiparación de conceptos no equivalentes, que puede incidir en que los estudiantes consideren que si existe correlación positiva ésta sea debida a una dependencia lineal (Sánchez Cobo, 1999). También se presentan descripciones incompletas de procedimientos, así como no diferenciar convenientemente las dos rectas de regresión, pudiendo derivar en que el estudiante tenga dificultad en diferenciar la variable explicativa de la explicada (Sánchez Cobo, 1999).

En otros casos, las relaciones que se establecen entre la recta de regresión y el coeficiente de correlación debiesen ser reflexionadas en clase por el profesor, pues 
podrían llevar a resultados equivocados y conclusiones erróneas. Este es el caso de la relación entre el coeficiente de correlación y la recta de regresión para establecer su aproximación, indicando que con una misma recta se pueden realizar predicciones para cada una de las variables si el valor del coeficiente de correlación lineal está próximo a $10-1$.

\section{Agradecimientos.}

Proyecto EDU2013-41141-P (MEC) y grupo FQM126 (Junta de Andalucía).

\section{Referencias}

Batanero, C., Estepa, A., \& Godino, J. D. (1997). Evolution of students' understanding of statistical association in a computer based teaching environment. En J. B. Garfield, \& G. Burrill (Eds.), Research on the role of technology in teaching and learning statistics (pp. 191-205). Voorburg, The Netherlands: Internacional Statistical Institute.

Cobo, B., \& Batanero, C. (2004). Significados de la media en los libros de texto de secundaria. Enseñanza de las Ciencias, 22(1), 5-18.

Cook, T. D., \& Reichardt, C. S. (2000). Métodos cualitativos y cuantitativos en investigación evaluativa. Paideia.

Cordero, F., \& Flores, R. (2007). El uso de las gráficas en el discurso matemático escolar. Un estudio socio epistemológico en el nivel básico a través de los libros de texto. Revista Latinoamericana de Matemática Educativa, 10(1), 7-38.

Chevallard, Y. (1991). La transposición didáctica. Del saber sabio al saber enseñado. Buenos Aires: Aique.

D’Amore, B. \& Godino, J. D. (2007). El enfoque ontosemiótico como un desarrollo de la teoría antropológica en didáctica de la matemática. Revista Latinoamericana de Investigación en Matemática Educativa, 10(2), 191-218.

Díaz-Levicoy, D. (2014). Un estudio empírico de los gráficos estadísticos en libros de texto de educación Primaria española. Tesis de Máster. Universidad de Granada.

Duval, R. (1993). Semiosis et Noesis. Lecturas en didáctica de la matemática: Escuela Francesa. México: Sección de Matemática Educativa del CINVESTAV-IPN.

Engel, J., \& Sedlmeier, P. (2011). Correlation and regression in the training of teachers. En C. Batanero, G. Burrill, \& C. Reading (Eds.), Teaching statistics in school mathematicschallenges for teaching and teacher education: A Joint ICMI/IASE study (pp, 247-258). New York: Springer.

Escolano, A. (2009). El manual escolar y la cultura profesional de los docentes. Tendencias Pedagógicas, 14, 169-180.

Estepa, A. (1994). Concepciones iniciales sobre la asociación estadística y su evolución como consecuencia de una enseñanza basada en el uso de ordenadores. Tesis doctoral. Universidad de Granada.

Estepa, A. (2008). Interpretación de los diagramas de dispersión por estudiantes de Bachillerato. Enseñanza de las Ciencias 26(2), 257-270.

Estepa, A., \& Batanero, C. (1995). Concepciones iniciales sobre la asociación estadística. Enseñanza de las Ciencias, 13(2), 155-170.

Fan, L., \& Zhu, Y. (2007). Representation of problem-solving procedures: A comparative look at China, Singapore, and US mathematics textbooks. Educational Studies in Mathematics, 
$66(1), 61-75$.

Font, V., Godino, J. D., \& D’Amore, B. (2007). An onto-semiotic approach to representations in mathematics education. For the Learning of Mathematics, 27(2), 2-7.

Gea, M. M., Batanero, C., Arteaga, P., Cañadas, G.R., \& Contreras, J. M. (2014). Análisis del lenguaje sobre la correlación y regresión en libros de texto de bachillerato. SUMA, 76, 3745.

Gea, M. M., Batanero, C., Cañadas, G. R., \& Contreras, J.M. (2013). Un estudio empírico de las situaciones-problema de correlación y regresión en libros de texto de bachillerato. En A. Berciano, G. Gutiérrez, A. Estepa, \& N. Climent (Eds), Investigación en Educación Matemática XVII (pp. 293-300). Bilbao: Sociedad Española de Investigación en Educación Matemática.

Gea, M. M., Batanero, C., Fernandes, J. A., \& Gómez, E. (2014). La distribución de datos bidimensionales en los libros de texto de matemáticas de Bachillerato. Quadrante, XXIII(2), 147-172.

Godino, J. D. (2002). Un enfoque ontológico y semiótico de la cognición matemática. Recherches en Didactique des Mathématiques, 22(2-3), 237-284.

Godino, J. D. (2012). Origen y aportaciones de la perspectiva ontosemiótica de investigación en Didáctica de la Matemática. En A. Estepa, A. Contreras, J. Deulofeu, M. C. Penalva, F. J. García, \& L. Ordóñez (Eds.), Investigación en Educación Matemática XVI (pp. 49 - 68). Jaén: Sociedad Española de Investigación en Educación Matemática.

Godino, J. D., \& Batanero, C. (1994). Significado institucional y personal de los objetos matemáticos. Recherches en Didactique des Mathématiques, 14(3), 325-355.

Godino, J. D., \& Batanero, C. (1999). Funciones semióticas en la enseñanza y aprendizaje de las matemáticas. En I. Vale, \& J. Portela (Eds.). Actas de la IX Reunión de la Sociedad Portuguesa de Investigación en Educación Matemática (SIEM) (pp. 47-62). Guimaraes (Portugal): Sociedad Portuguesa de Profesores de Matemáticas.

Godino, J. D., Batanero, C., \& Font, V. (2007). The onto-semiotic approach to research in mathematics education. ZDM. The International Journal on Mathematics Education, 39(1-2), 127-135.

Godino, J. D., Font, V., Contreras, A., \& Wilhelmi, M. R. (2006). Una visión de la didáctica francesa desde el enfoque ontosemiótico de la cognición e instrucción matemática. Revista Latinoamericana de Investigación en Matemática Educativa, 9(1), 117-150.

Herbel, B. A. (2007). From intended curriculum to written curriculum: Examining the" voice" of a mathematics textbook. Journal for Research in Mathematics Education, 38(4), 344369.

Lavalle, A. L., Micheli, E. B., \& Rubio, N. (2006). Análisis didáctico de regresión y correlación para la enseñanza media. RELIME, 9(3), 383-406.

Martínez, M. (2006). La investigación cualitativa (síntesis conceptual). Revista de Investigación en Psicología, 9(1), 123-146.

Mayén, S. (2009). Comprensión de las medidas de tendencia central en estudiantes mexicanos de Educación Secundaria y Bachillerato. Tesis doctoral. Universidad de Granada.

M.E.C. (2007). Real Decreto 1467/2007, de 2 de noviembre, por el que se establece la estructura de bachillerato y se fijan sus enseñanzas mínimas. Madrid: Autor.

M.E.C.D. (2014). Real Decreto 1105/2014, de 26 de diciembre, por el que se establece el currículo básico de la Educación Secundaria Obligatoria y del Bachillerato. Madrid: Autor.

Noguero, F. L. (2002). El análisis de contenido como método de investigación. Revista de 
Educación, 4, 167-180.

Reys, B. J., Reys, R. E., \& Chavez, O. (2004). Why mathematics textbooks matter. Educational Leadership, 61(5), 61-66.

Ruiz, B. (2013). Un análisis epistemológico y cognitivo de la variable aleatoria. Tesis doctoral. Universidad de Granada.

Sánchez Cobo, F.T. (1999). Significado de la correlación y regresión para los estudiantes universitarios. Tesis doctoral. Universidad de Granada.

Sánchez Cobo, F. T., Estepa, A., \& Batanero, C. (2000). Un estudio experimental de la estimación de la correlación a partir de diferentes representaciones. Enseñanza de las Ciencias, 18(2), 297-310.

Shield, M., \& Dole, S. (2013). Assessing the potential of mathematics textbooks to promote deep learning. Educational Studies in Mathematics, 82(2), 183-199.

Truran (1995). Some undergraduates' understanding of the meaning of a correlation coefficient, en B. Atweh, y S. Clavel (Eds.), Proceedings of the Eigteenth Annual Conference of the Mathematics Education Research Group of Australasia (MERGA) (pp. 524-529). Darwin, Australia: Northern Territory University.

Zieffler, A., \& Garfield, J. (2009). Modeling the growth of students' covariational reasoning during an introductory statistics course. Statistics Education Research Journal, 8(1), 7-31.

\section{Anexo 1: Textos utilizados en el análisis}

[H1] Colera, J., Oliveira, M J., García, R., \& Santaella, E. (2008). Matemáticas aplicadas a las Ciencias Sociales I. Madrid: Grupo Anaya.

[H2] Arias, J. M., \& Maza, I. (2011). Matemáticas aplicadas a las Ciencias Sociales 1. Madrid: Grupo Editorial Bruño.

[H3] Anguera, J., Biosca, A., Espinet, M. J., Fandos, M. J., Gimeno, M., \& Rey, J. (2008). Matemáticas I aplicadas a las Ciencias Sociales. Barcelona: Guadiel.

[H4] Monteagudo, M. F. \& Paz, J. (2008). $1^{\circ}$ Bachillerato. Matemáticas aplicadas a las Ciencias Sociales. Zaragoza: Luis Vives.

[H5] Martínez, J. M., Cuadra, R., \& Heras, A. (2008). Matemáticas aplicadas a las Ciencias Sociales. $1^{\circ}$ Bachillerato. Madrid: McGraw-Hill.

[H6] Bescós, E. \& Pena, Z. (2008). Matemáticas aplicadas a las Ciencias Sociales. $1^{\circ}$ Bachillerato. Vizcaya: Oxford University Press.

[H7] Antonio, M., González, L., Lorenzo, J., Molano, A., del Río, J., Santos, D., \& de Vicente, M. (2009). Matemáticas aplicadas a las Ciencias Sociales I. Madrid: Santillana Educación.

[H8] Vizmanos, J. R., Hernández, J., Alcaide, F., Moreno, M., \& Serrano, E. (2008). Matemáticas aplicadas a las Ciencias Sociales 1. Madrid: Grupo SM.

[T1] Colera, J., Oliveira, M. J., García, R., \& Santaella, E. (2008). Matemáticas I. Madrid: Grupo Anaya.

[T2] Arias, J. M., \& Maza, I. (2011). Matemáticas 1. Madrid: Grupo Editorial Bruño.

[T3] Biosca, A., Doménech, M., Espinet, M. J., Fandos, M. J., \& Jimeno, M. (2008). Matemáticas I. Barcelona: Guadiel - Grupo Edebé.

[T4] Monteagudo, M. F., \& Paz, J. (2008). $1^{\circ}$ Bachillerato. Matemáticas. Ciencias y Tecnología. Zaragoza: Edelvives (Editorial Luis Vives).

[T5] Martínez, J. M., Cuadra, R., \& Barrado, F. J. (2007). Matemáticas $1^{\circ}$ Bachillerato. Madrid: McGraw-Hill. 
[T6] Bescós, E., \& Pena, Z. (2009). Matemáticas. $1^{\circ}$ Bachillerato. Navarra: Oxford University Press España.

[T7] Antonio, M., González, L., Lorenzo, J., Molano, A., del Río, J., Santos, D., \& y de Vicente, M. (2008). Matemáticas $1^{\circ}$ Bachillerato. Madrid: Santillana Educación.

[T8] Vizmanos, J. R., Hernández, J., Alcaide, F., Moreno, M., \& Serrano, E. (2008). Matemáticas 1. Madrid: Ediciones SM.

\section{Referencias a los autores}

María Magdalena Gea Serrano, Universidad de Granada (España).mmgea@ugr.es

María del Mar López-Martín, Universidad de Granada (España).

mariadelmarlopez@ugr.es

Rafael Roa Guzmán, Universidad de Granada (España).rroa@ugr.es 


\title{
Semiotic conflicts on the correlation and regression textbooks High School.
}

\author{
María M. Gea Serrano, Universidad de Granada (España) \\ María del Mar López-Martín, Universidad de Granada (España) \\ Rafael Roa Guzmán, Universidad de Granada (España)
}

Correlation and regression are fundamental statistical ideas, due to its usefulness to model phenomena in different fields, but previous research has described several misconceptions in understanding these topics. To complement previous research on the presentation of correlation and regression in the textbooks, the aim of this papr is to characterize the semiotic conflicts implicit or explicit in the presentation in Spanish High School textbooks and that may influence the acquisition of them by the students.

We base on the onto-semiotic approach of mathematical knowledge and instruction in mathematics education that discriminates between institutional and personal meaning of mathematical objects; semiotic conflict in this framework is defined as a disparity between these two meanings. A content analysis of the chapters dealing with this topic is performed on a sample of 16 high school textbooks, eight of the Science and Technology specialty and eight directed to Humanities and Social Sciences. With an inductive and cyclic procedure we identified and classified the main types of conflicts concerning the language used, the definition of concepts and presentation of properties and procedures, and the conflicts implicit in some arguments.

Our results show a wide variety of these potential conflicts in any of the above categories, all the books and both specialties. These results may serve to alert the teacher of their existence, help them complement the presentation of the theme in the textbooks and improve the student learning. Finally, some criteria are provided to enhance the presentation of correlation and regression in the High School textbooks. 